\title{
REFLECTION OF HIGH-FREQUENCY PHONONS AT SILICON-SOLID INTERFACES
}

\author{
D. MARX and W. EISENMENGER \\ Universität Stuttgart, Physikalisches Institut, 7000 Sturtgart 80, West Germany
}

Received 3 December 1980

Revised manuscript received 10 February 1981

\begin{abstract}
In reflection experiments with phonons of frequencies above $280 \mathrm{GHz}$ propagating along (110) directions we observed large deviations from the acoustic mismatch theory for silicon-metal, silicon-condensed gas, and silicon-liquid helium interfaces.
\end{abstract}

In quantitative phonon generation and detection experiments with superconducting tunneling junctions Trumpp measured excessive $2 \Delta$-phonon losses of 90 to $98 \%$, as compared with the predictions of the acoustic mismatch model [1-3]. He located the main loss source at the boundaries between the junctions and the substrate.

This phonon loss considerably reduces the sensitivity in phonon spectroscopy experiments $[4,5]$ As it is related to the well-known anomaly of the Kapitza resistance between solids and helium [6,7], this phonon loss motivated the present reflection experiments, avoiding the limitation that the metal film is part of a tunneling junction.

The experimental set-up is similar to the one we described previously [3], when we discussed the phonon reflection at the free silicon surface. By taking the phonon focusing in silicon into account we were able to separate diffusively and specularly reflected phonons by their propagation time, frequency dependence, and propagation paths (fig. 1).

We scrutinized phonon reflection at the siliconmetal interfaces by comparing it with the reflection at the free silicon surface (fig. 2). In this way, we determined the ratio $R_{i}^{\exp }$ of the pulse amplitude of phonons with polarization $i$ reflected at the covered part of the crystal surface to that of the uncovered surface part. This experimental reflection coefficient is compared with the value $R_{i}^{\text {theor }}$, as calculated by the acoustic mismatch theory [8]. The high-purity Si-substrate crystals are mechanically, chemically, or sputter polished. Various superconducting or normal-conducting interface-forming metals are evaporated or sputter-deposited by Ar-beam technique. The film thickness is chosen to be large compared to the phonon mean free path so that backscattering from the free metal surface gives only negligible contributions to the detector signal, as tested by an additional He coverage of the metal film (see fig. 1). We use constantan heaters and $\mathrm{Sn}$ tunneling junctions as generators, Sn tunneling junctions as detectors.

The results of our reflection measurements for different layers on Si are shown in table 1 . They may be summarized as follows:

(i) For all metals (theoretically good acoustic match) the experimental reflection coefficients are higher than the calculated values.

(ii) For condensed solid gases (poor acoustic match) the measured reflection coefficients are smaller than the calculated values.

(iii) The variation of the experimental reflection coefficients for repeated deposition experiments of the same metal is much larger than the measuring accuracy and is similar in magnitude to the variation for different materials.

(iv) The reduction in the detector signal caused by condensed gases is strongest for the diffusively scattered phonons.

In conclusion: in our experiments we find for all solid-solid interfaces a reflection coefficient deviating 


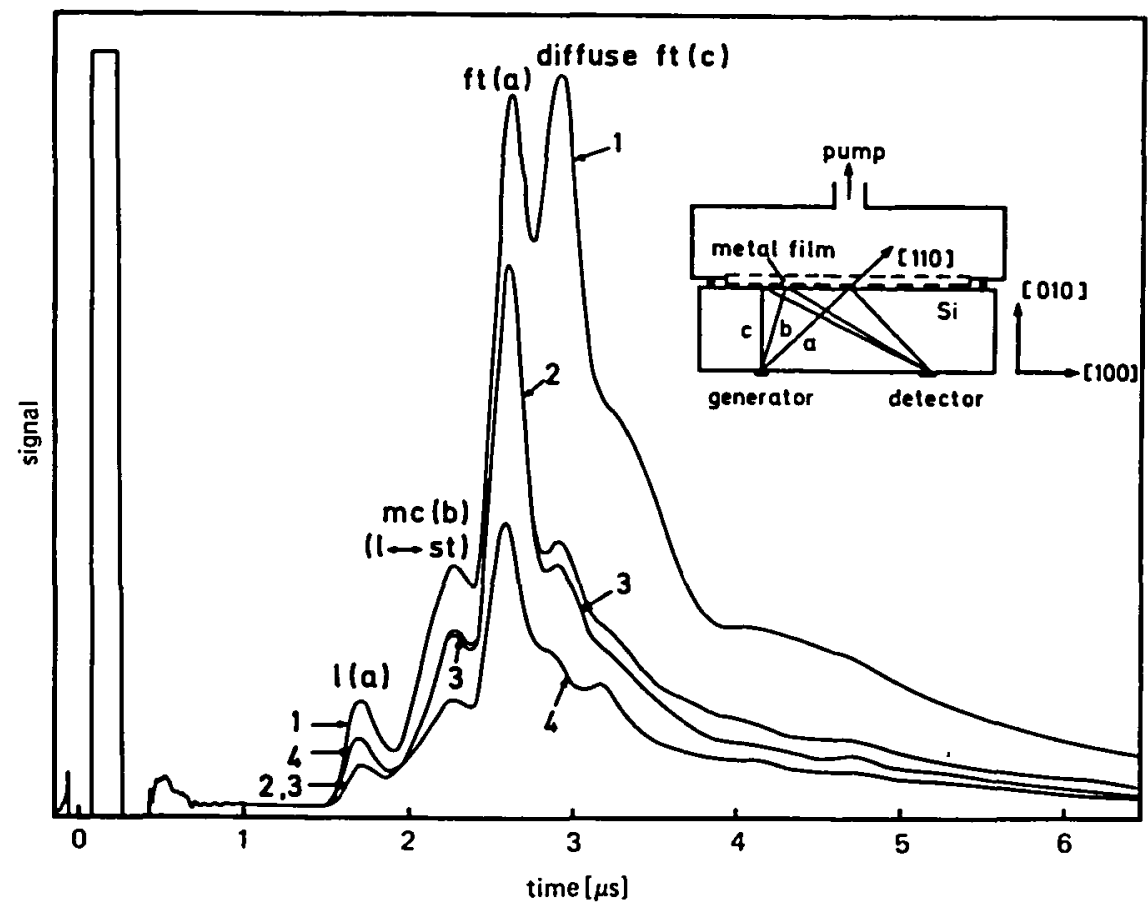

Fig. 1. Typical detector signals for the scattering surface in vacuum (1), covered with $\mathrm{He}(4)$, a $280 \mathrm{~nm}$ thick $\mathrm{Cr}$ film (2), and $\mathrm{Cr}$ film plus $\mathrm{He}(3)$. Pulse duration $100 \mathrm{~ns}$, bath temperature $1.37 \mathrm{~K}$. The inset shows the experimental set-up and the propagation paths of the individual pulses. Specularly reflected phonons: longitudinal $(\ell)$, mode converted (mc), and fast transverse (ft) phonons. The fourth pulse is caused by diffusively scattered fast transverse phonons.

from the acoustic mismatch model. The reflection of the phonons is almost independent of the covering material and ranges from 20 to $70 \%$ for longitudinal phonons.

From this it follows that the ideal interface condi-

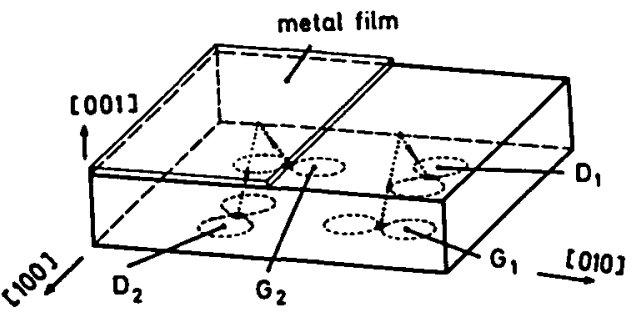

Fig. 2. Si crystal with two generator-detector pairs, simultaneously prepared, for reflection experiments at $\mathrm{Si}$-metal interfaces. Opposite to one pair the scattering surface is covered with the metal film, opposite to the other the scattering surface is in vacuum. tions of the acoustic mismatch theory are not realized in our experiments. Obviously, the observed strong diffusive scattering indicates that the interface is not flat compared to the phonon wavelength of $\leqslant 20 \mathrm{~nm}$ and that, in addition, weakly bound atoms, molecules, etc., lead to diffusive elastic phonon scattering [9]. The weak adhesion of the metal films on the Si surface (weak adhesion compared with Van der Waals forces, but the films cannot be removed by an adhesive tape) indicates that the continuity condition of the displacement vector at the interface may be violated for large phonon wave vectors [10].

As is known from heat conduction experiments $[6,7]$, the acoustic mismatch theory satisfactorily describes the thermal boundary resistance between solids in the temperature range below $0.1 \mathrm{~K}$, i.e. for large phonon wavelengths. In our experiments, in contrast, we are limited to phonon frequencies above $280 \mathrm{GHz}$ by the high-pass properties of the tin tun. neling junction detectors. 
Table 1

Calculated and measured values for the ratio $R_{i}$. Above the measured values the preparation of the substrate surface (mp, $\mathrm{cp}$, ip: mechanical, chemical, sputter polish) and the thickness of the metal films are indicated.

\begin{tabular}{|c|c|c|c|c|c|c|}
\hline \multirow{2}{*}{ Material } & \multirow[t]{2}{*}{ Calculated ratio $R_{i}^{\text {theor }}$} & \multicolumn{5}{|c|}{ Measured ratio $R_{i}^{\exp }$} \\
\hline & & $\begin{array}{l}\mathrm{mp} \\
400 \mathrm{~nm}\end{array}$ & $\begin{array}{l}\mathrm{mp} \\
630 \mathrm{~nm}\end{array}$ & $\begin{array}{l}\mathrm{cp} \\
1000 \mathrm{~nm}\end{array}$ & ip $1000 \mathrm{~nm}$ & $\begin{array}{l}\text { cp, ip a) } \\
>600 \mathrm{~nm}\end{array}$ \\
\hline & $R_{Q} 0.002$ & 0.25 & 0.64 & 0.60 & 0.56 & 0.26 \\
\hline & $R_{\mathrm{mc}}$ no calculated value & 0.37 & 0.51 & 0.56 & 0.69 & 0.51 \\
\hline & $R_{\mathrm{ft}} 0.01$ & 0.34 & 0.54 & 0.41 & 0.76 & 0.39 \\
\hline & $R_{\mathrm{d}}$ no calculated value & 0.38 & 0.31 & 0.38 & 0.48 & 0.39 \\
\hline
\end{tabular}

$\Delta 1$

$$
\begin{aligned}
& R_{\mathrm{Q}} 0.03 \\
& R_{\mathrm{mc}} \text { no calculated value } \\
& R_{\mathrm{ft}} 0.01 \\
& R_{\mathrm{d}} \text { no calculated value }
\end{aligned}
$$

In

$$
\begin{aligned}
& R_{\mathrm{Q}} 0.04 \\
& R_{\mathrm{mc}} \text { no calculated value } \\
& R_{\mathrm{ft}} 0.003 \\
& R_{\mathrm{d}} \text { no calculated value }
\end{aligned}
$$

Con-

stantan

$$
\begin{aligned}
& R_{\mathrm{Q}} 0.06 \\
& R_{\mathrm{mc}} \text { no calculated value } \\
& R_{\mathrm{ft}} 0.15 \\
& R_{\mathrm{d}} \text { no calculated value }
\end{aligned}
$$

Cr

$R_{\ell} 0.04$

$R_{\text {mc }}$ no calculated value $R_{\mathrm{ft}} \mathbf{0 . 2 0}$

$R_{\mathrm{d}}$ no calculated value

$\mathrm{N}_{2}$

$$
\begin{aligned}
& R_{\mathrm{Q}} 0.75 \\
& R_{\mathrm{mc}} \text { no calculated value } \\
& R_{\mathrm{ft}} 0.69 \\
& R_{\mathrm{d}} \text { no calculated value }
\end{aligned}
$$

$\mathrm{Ne}$

$$
\begin{aligned}
& R_{\mathrm{Q}} 0.87 \\
& -R_{\mathrm{mc}} \text { no calculated value } \\
& R_{\mathrm{ft}} 0.60 \\
& R_{\mathrm{d}} \text { no calculated value }
\end{aligned}
$$

Ar

$$
\begin{aligned}
& R_{\mathrm{Q}} 0.69 \\
& R_{\mathrm{mc}} \text { no calculated value } \\
& R_{\mathrm{ft}} 0.57 \\
& R_{\mathrm{d}} \text { no calculated value }
\end{aligned}
$$

He

$$
\begin{aligned}
& R_{Q} 0.99 \\
& R_{\text {mc }} \text { no calculated value } \\
& R_{\mathrm{ft}} 1.00 \\
& R_{\mathrm{d}}
\end{aligned}
$$

$\mathrm{mp}$ $200 \mathrm{~nm}$

0.26

0.56

0.40

0.42

mp $800 \mathrm{~nm}$

0.41

0.38

0.26

0.25

$\mathrm{mp}$

$100 \mathrm{~nm}$

0.81

0.63

0.74

0.42

$\mathrm{mp}$

$280 \mathrm{~nm}$

0.41

0.81

0.74

0.42

$\mathrm{mp}$

0.47
0.46

\begin{tabular}{|c|c|}
\hline $\mathrm{mp}$ & $p$ \\
\hline 0.43 & 0.45 \\
\hline 0.49 & 0.44 \\
\hline 0.39 & 0.40 \\
\hline$<0.27$ & $<0.25$ \\
\hline
\end{tabular}

$\approx 0.45$

mp b)

0.63

0.50

0.47

$<0.37$

ip

0.43

0.45

0.39

$<0.25$

mp

0.67

0.44

0.40

$<0.20$

$m p \quad m p$

$1600 \mathrm{~nm} \quad 1100 \mathrm{~nm}$

$\approx 0.4 \quad \approx 0.43$

$m p$ b)

$145 \mathrm{~nm}$

0.56

0.66

0.65

0.34

$$
0.47
$$$$
<0.23
$$

a) Metal film sputter deposited by Ar-beam technique. b) Generator was a tin tunneling junction. 
From phonon reflection experiments $[11]$ it is further known, that the acoustic mismatch theory gives a good description for the thermal boundary resistance of solid-liquid helium interfaces for high phonon frequencies if the surface is atomically flat and without any contamination, i.e. prepared by cleaving at low temperature $(\leqslant 4 \mathrm{~K})$. The smallest contamination of the surface by $\mathrm{N}_{2}$ molecules, for example, or disturbance of the flatness by cleavage steps, e.g., give rise to additional phonon scattering.

Since in phonon spectroscopy it is generally impractical to prepare substrate surfaces and metal films under similar ideal conditions, scattering centers and interface imperfections cannot be avoided. To optimize the phonon yield one should, therefore, try to improve the coupling of the elastically scattered phonons to the metal film by other means. The observed differences in the coupling, i.e. the large variation of the $R^{\exp }$ values in repeated experiments with the same interfaceforming material, are in this respect indicative for a possible increase of the phonon yield by improved deposition procedures.
The authors gratefully acknowledge financial support by the "Deutsche Forschungsgemeinschaft" and valuable discussions with $K$. Lassmann, $O$. Koblinger and M. Welte. We would like to thank Mrs. G. Mayerhöffer for preparing the substrates.

\section{References}

[1] H.J. Trumpp, P.W. Epperlein and K. Lassmann, J. de Phys. C4, Suppl. to No. 10 (1972) C-29.

[2] H.J. Trumpp, K. Lassmann and W. Eisenmenger, Phys. Lett. $41 \mathrm{~A}$ (1972) 431.

[3] D. Marx, J. Buck, K. Lassmann and W. Eisenmenger, J. de Phys. C6, Suppl. to No. 8 (1978) C6-1015.

[4] H. Kinder, Phys. Rev. Lett. 28 (1972) 1564.

[5] W. Forkel, M. Welte and W. Eisenmenger, Phys. Rev. Lett. 31 (1973) 215.

[6] L.J. Challis, J. Phys. C7 (1974) 481.

[7] A.C. Anderson, 2nd Intern. Conf. on Phonon scattering in solids (Nottingham, 1975).

[8] B.A. Auld, Acoustic waves in solids (Wiley, 1973).

[9] H.J. Trumpp and W. Eisenmenger, Z. Phys. B28 (1977) 159.

[10] Ch. Steinbrüchel, Z. Phys. B24 (1976) 293.

[11] J. Weber, W. Sandmann, W. Dietsche and H. Kinder, Phys. Rev. Lett. 40 (1978) 1469. 